doi: $10.13108 / 2019-11-4-140$

\title{
THRESHOLD PHENOMENON FOR A FAMILY OF THE GENERALIZED FRIEDRICHS MODELS WITH THE PERTURBATION OF RANK ONE.
}

\author{
S.N. LAKAEV, M. DARUS, S.T. DUSTOV
}

\begin{abstract}
In this work we consider a family $H_{\mu}(p), \mu>0, p \in \mathbb{T}^{3}$, of the generalized Friedrichs models with the perturbation of rank one. This system describes a system of two particles moving on the three dimensional lattice $\mathbb{Z}^{3}$ and interacting via a pair of local repulsive potentials. One of the reasons to consider such family of the generalized Friedrichs models is that this family generalizes and involves some important behaviors of the Hamiltonians for systems of both bosons and fermions on lattices. In the work, we study the existence or absence of the eigenvalues of the operator $H_{\mu}(p)$ located outside the essential spectrum depending on the values of $\mu>0$ and $p \in U_{\delta}\left(p_{0}\right) \subset \mathbb{T}^{3}$. We prove a analytic dependence on the parameters for such eigenvalue and an associated eigenfunction and the latter is found in a certain explicit form. We prove the existence of coupling constant threshold $\mu=\mu(p)>0$ for the operator $H_{\mu}(p), p \in U_{\delta}\left(p_{0}\right)$, namely, we show that the operator $H_{\mu}(p)$ has no eigenvalue for all $0<\mu<\mu(p)$ and there exists a unique eigenvalue $z(\mu, p)$ for each $\mu>\mu(p)$ and this eigenvalue is located above the threshold $z=M(p)$. We find necessary and sufficient conditions allowing us to determine whether the threshold $z=M(p)$ is an eigenvalue or a virtual level or a regular point in the essential spectrum of the operator $H_{\mu}(p), p \in U_{\delta}\left(p_{0}\right)$.
\end{abstract}

Keywords: coupling constant threshold, repulsive potential, eigenvalue, generalized Friedrichs model, regular point.

Mathematics Subject Classification: 35P15, 47A10

\section{INTRODUCTION}

In the present paper we consider a family of generalized Friedrichs models with rank one perturbations $H_{\mu}(p), \mu>0, p \in U_{\delta}\left(p_{0}\right) \subset \mathbb{T}^{3}$, where $U_{\delta}\left(p_{0}\right)$ is a $\delta$-neighborhood of the point $p=p_{0} \in \mathbb{T}^{3}$. This family describes a system of two particles on the three-dimensional lattice $\mathbb{Z}^{3}$ interacting via a pair of local repulsive potentials.

We show that if the parameters of the generalized Friedrichs model satisfy some conditions, there exists a coupling constant threshold $\mu=\mu(p)>0$ such that the operator $H_{\mu}(p), p \in$ $U_{\delta}\left(p_{0}\right)$ has no eigenvalue for all $0<\mu<\mu(p)$ and at the same time, it possesses a unique eigenvalue $z(\mu, p)$ above the threshold $z=M(p)$ of the essential spectrum for each $\mu>\mu(p)$. We find a certain explicit form for an associated eigenfunction and prove its analytic dependence on the parameters. We also find necessary and sufficient conditions determining whether the threshold $z=M(p)$ is an eigenvalue or a virtual level or a regular point in the essential spectrum of $H_{\mu}(p), p \in U_{\delta}\left(p_{0}\right)$.

S.N.LakaEv, M.Darus, S.T.Dustov, Threshold Phenomenon for a Family of the Generalized FRIEDRICHS MODELS WITH THE PERTURBATION OF RANK ONE.

(c) S.N.Lakaev, M.Darus, S.T.Dustov 2018.

The work was supported by the Fundamental Science Foundation of Uzbekistan(grant no. OT-F4-66) AND BY THE GRANT NO. GUP-2019-032.

Submitted November 6, 2018. 
In [1] for a family of the generalized Friedrichs models $H_{\mu}(p), \mu>0, p \in \mathbb{T}^{2}$ either the existence or absence of a positive coupling constant threshold $\mu=\mu(p)>0$ depending on the parameters of the model has been proved.

One of the reasons to consider the family of the generalized Friedrichs models interacting via pair local repulsive potentials is as follows: this family generalizes and involves some important behaviors featured by the Schrödinger operators associated with the Hamiltonian of the systems of two arbitrary particles moving on $\mathbb{R}^{d}$ or $\mathbb{Z}^{d}, d \geqslant 1$, as well as with the Hamiltonian for systems of both bosons and fermions [1], [2], [3, [4].

Moreover, as it was stated in [5], [6], physics stable composite objects are usually formed by attractive forces, which allow the constituents to lower their energy by binding together. The repulsive forces separates particles in free space. However, in structured environment such as a periodic potential and in the absence of dissipation, stable composite objects can exist even for repulsive interactions.

The family of the generalized Friedrichs models theoretically adequately describes this phenomenon relating to repulsive forces since the two-particle discrete Schrödinger operators with rank one interactions are a special case of this family.

The generalized Friedrichs model, i.e., the case, where the non-perturbed operator $H_{0}$ is a multiplication operator by arbitrary function with Van Hove singularities (critical points) defined on the closed interval $[a, b]$ was considered in [3]. In this case, unlike to usual Friedrichs model, the multiplicity of continuous spectrum of the non-perturbed operator is not constant.

The generalized Friedrichs models appear mostly in the problems of solid state physics [8], [9], quantum mechanics [10], and quantum field theory [11], [12] and in general settings have been studied in [2], [3, [7].

In [13], the family of generalized Friedrichs models with rank one perturbations $H_{\mu}(p), \mu>0$, $p \in(-p, p]^{3}$, associated with a system of two particles on the three-dimensional lattice $\mathbb{Z}^{3}$ was considered. In a special case of multiplication operator and under the assumption that the operator $H_{\mu}(0), 0 \in \mathbb{T}^{3}$ has a coupling constant threshold $\mu_{0}(0)>0$, the existence of a unique eigenvalue below the bottom of the essential spectrum of $H_{\mu_{0}(0)}(p), p \in(-p, p]^{3}$ for all nontrivial values of $p \in \mathbb{T}^{3}$ was proved.

In [14, an expansion for an eigenvalue $E(\mu, p)$ of $H_{\mu}(p), \mu>0, p \in(-p, p]$ was found in some neighborhood of the point $\mu=\mu(p) \geqslant 0$. In [4], for a special family of the generalized Friedrichs models, the existence of eigenvalues was proved for some values of quasi-momentum $p \in \mathbb{T}^{d}$ in a neighborhood of $p_{0} \in \mathbb{T}^{d}$.

In celebrated work [15], B.Simon and M.Klaus considered a family of Schrödinger operators $H=-\Delta+\mu V$ in a situation, where as $\mu$ tended to $\mu_{0}$, some eigenvalue $e_{i}(\mu)$ tended to 0 , i.e., this eigenvalue was absorbed by the essential spectrum, and vice versa, as $\mu$ tended to from the right $\mu_{0}$, a new eigenvalue emerged from the essential spectrum. In [15], this phenomenon was called coupling constant threshold.

In [16] the Hamiltonian of a system of two identical quantum mechanical particles (bosons) moving on the $d$-dimensional lattice $\mathbb{Z}^{d}, d \geqslant 3$ and interacting via a pair zero-range repulsive pair potentials was considered. For the associated two-particle Schrödinger operator $H_{\mu}(K)$, $K \in \mathbb{T}^{d}=(-\pi, \pi]^{d}$, the existence of coupling constant threshold $\mu=\mu_{0}(K)>0$ was proven: the operator had no eigenvalue for all $0<\mu<\mu_{0}$, but for each $\mu>\mu_{0}$, it possessed a unique eigenvalue $z(\mu, K)$ above the upper edge of the essential spectrum of $H_{\mu}(K)$. An asymptotics for $z(\mu, K)$ was found as $\mu \rightarrow \mu_{0}(K)$ and $K \rightarrow 0$.

In [17], for a wide class of two-body energy operators $H_{2}(k)$ on the $d$-dimensional lattice $\mathbb{Z}^{d}$, $d \geq 3, k$ being the two-particle quasi-momentum, it was proved that for all nontrivial values $k \neq 0$, the discrete spectrum of $H_{2}(k)$ below its threshold is non-empty provided the following two assumptions (i) and (ii) are satisfied: 
(i) the operator $H_{2}(0)$ has either an eigenvalue or a virtual level at the bottom of its essential spectrum

(ii) the one-particle free Hamiltonian in the coordinate representation generates positivity preserving semi-group.

In [15] the existence of a coupling constant threshold was assumed, while time in [16] the coupling constant threshold was determined by the given data of the Hamiltonian.

We note that for the Hamiltonian of a system of two identical particles moving on $\mathbb{R}^{2}$ or $\mathbb{Z}^{2}$ the coupling constant threshold vanishes if the particles are bosons and the coupling constant threshold is positive if particles are fermions.

\section{PRELIMINARIES AND MAIN RESUltS}

Let $\mathbb{Z}^{3}$ be the three-dimensional hypercubes lattice and $\mathbb{T}^{3}=(-\pi, \pi]^{3}$ be the threedimensional torus (Brillouin zone), the dual group of $\mathbb{Z}^{3}$. The operators of addition and multiplication by number of the elements of torus $\mathbb{T}^{3} \equiv(-\pi, \pi]^{3} \subset \mathbb{R}^{3}$ was defined as operations in $\mathbb{R}^{3}$ modulo $\left(2 \pi \mathbb{Z}^{3}\right)$.

Let $L^{2}\left(\mathbb{T}^{3}\right)$ be the Hilbert space of square-integrable functions defined on the torus $\mathbb{T}^{3}$ and $\mathbb{C}^{1}$ be the one-dimensional complex Hilbert space.

We consider a family of the generalized Friedrichs models acting in $L^{2}\left(\mathbb{T}^{3}\right)$ as follows:

$$
H_{\mu}(p)=H_{0}(p)+\mu \Phi^{*} \Phi, \quad \mu>0 .
$$

Here

$$
\begin{array}{ll}
\Phi: L^{2}\left(\mathbb{T}^{3}\right) \rightarrow \mathbb{C}^{1}, & \Phi f=(f, \varphi)_{L^{2}\left(\mathbb{T}^{3}\right)}, \\
\Phi^{*}: \mathbb{C}^{1} \rightarrow L^{2}\left(\mathbb{T}^{3}\right), & \Phi^{*} f_{0}=\varphi(q) f_{0},
\end{array}
$$

where $(\cdot, \cdot)_{L^{2}\left(\mathbb{T}^{3}\right)}$ is the scalar product in $L^{2}\left(\mathbb{T}^{3}\right)$ and $H_{0}(p), p \in \mathbb{T}^{3}$ is a multiplication operator by a function $w_{p}(\cdot):=w(p, \cdot)$, that is,

$$
\left(H_{0}(p) f\right)(q)=w_{p}(q) f(q), \quad f \in L^{2}\left(\mathbb{T}^{3}\right) .
$$

We observe that for each $f \in L^{2}\left(\mathbb{T}^{3}\right)$ and $g_{0} \in \mathbb{C}^{1}$ the identity

$$
\left(\Phi f, g_{0}\right)_{\mathbb{C}^{1}}=\left(f, \Phi^{*} g_{0}\right)_{L^{2}\left(\mathbb{T}^{3}\right)}
$$

holds.

In the paper, we make the following assumption.

Assumption 1. The following conditions are satisfied:

(i) the function $\varphi(\cdot)$ is nontrivial and real-analytic and has no singularities on the torus $\mathbb{T}^{3}$.

(ii) the function $w(\cdot, \cdot)$ is real-analytic function on $\left(\mathbb{T}^{3}\right)^{2}=\mathbb{T}^{3} \times \mathbb{T}^{3}$ and has a unique nondegenerated maximum at $\left(p_{0}, q_{0}\right) \in\left(\mathbb{T}^{3}\right)^{2}$.

Remark 1. We identify the real-analytic functions on $\mathbb{T}^{3}$ with the real-analytic functions on $\mathbb{R}^{3}$ being $2 \pi$-periodic in each variable.

The perturbation $v=\Phi^{*} \Phi$ is positive operator of rank one. Hence, by the well-known Weyl theorem [18], the essential spectrum fills the following segment on the real axis:

$$
\sigma_{\text {ess }}\left(H_{\mu}(p)\right)=\sigma_{\text {ess }}\left(H_{0}(p)\right)=[m(p), M(p)],
$$

where

$$
m(p)=\min _{q \in \mathbb{T}^{3}} w_{p}(q), \quad M(p)=\max _{q \in \mathbb{T}^{3}} w_{p}(q) .
$$

By Assumption 1, there exist a $\delta$-neighborhood $U_{\delta}\left(p_{0}\right) \subset \mathbb{T}^{3}$ of the point $p=p_{0} \in \mathbb{T}^{3}$ and an analytic vector function $\mathbf{q}_{0}: U_{\delta}\left(p_{0}\right) \rightarrow \mathbb{T}^{3}$ such that for each $p \in U_{\delta}\left(p_{0}\right)$, the point 
$\mathbf{q}_{0}(p)=\left(q_{0}^{(1)}(p), q_{0}^{(2)}(p), q_{0}^{(3)}(p)\right) \in \mathbb{T}^{3}$ is a unique non-degenerated maximum of the function $w_{p}(\cdot)$, see Lemma 3.1 . Moreover, the following integral

$$
\frac{1}{\mu(p)}=\int_{\mathbb{T}^{3}} \frac{\varphi^{2}(s) d s}{M(p)-w_{p}(s)}>0
$$

is well-defined, see Lemma 3.4 .

The positive number $\mu(p)>0$ is called coupling constant threshold.

Definition 2.1. The threshold $z=M(p)$ is called a regular point of the essential spectrum of the operator $H_{\mu}(p)$ if the equation $H_{\mu}(p) f=M(p) f$ has only trivial solution $f=0$ in $L^{2}\left(\mathbb{T}^{3}\right)$.

Let $L^{1}\left(\mathbb{T}^{3}\right)$ be the Banach space of integrable functions on $\mathbb{T}^{3}$.

Definition 2.2. The threshold $z=M(p)$ is called a $M(p)$ energy resonance (virtual level) in the essential spectrum of the operator $H_{\mu}(p)$ if the equation $H_{\mu}(p) f=M(p) f$ has a non-trivial solution $f \in L^{1}\left(\mathbb{T}^{3}\right) \backslash L^{2}\left(\mathbb{T}^{3}\right)$. The solution $f$ is called virtual state of the operator $H_{\mu}(p)$.

Remark 2. The set $\mathbb{G}$ of $\mu>0$, for which the threshold is a regular point of the essential spectrum $\sigma_{\text {ess }}\left(H_{\mu}(p)\right)$ of $H_{\mu}(p)$, is an open set in $(0,+\infty)$. More precisely, $\mathbb{G}=(0,+\infty) \backslash\{\mu(p)\}$.

Remark 3. If the threshold $z=M(p)$ is a regular point of $H_{\mu}(p)$, then the number of eigenvalues of the operator $H_{\mu}(p)$ above the threshold $M(p)$ does not change under small perturbations of $\mu \in \mathbb{G}$, see Statements (i), (ii) and (iii) of Theorem 2.1.

Next theorem provides a necessary and sufficient conditions for existence of a unique eigenvalue $E(\mu, p)$ above the threshold of the essential spectrum of $H_{\mu}(p), p \in U_{\delta}\left(p_{0}\right)$. We prove that for a fixed $p \in U_{\delta}\left(p_{0}\right)$ the function $E(\cdot, p)$ is analytic in $(\mu(p),+\infty)$. Moreover, for the associated eigenfunction we find an explicit expression and prove its analyticity. Furthermore, in the case $\mu=\mu(p)>0$, we prove that the threshold $M(p)$ of the essential spectrum is either a virtual level or eigenvalue for the operator $H_{\mu}(p), p \in \mathbb{T}^{3}$.

Theorem 2.1. Let Assumption 1 hold and $p \in U_{\delta}\left(p_{0}\right)$. Then the following statements are true:

(i) The operator $H_{\mu}(p)$ has a unique eigenvalue $E(\mu, p)$ above the threshold $M(p)$ of the essential spectrum if and only if $\mu>\mu(p)$. The function $E(\cdot, p)$ is a monotonically increasing real-analytic function on the interval $(\mu(p),+\infty)$ and the function $E(\mu, \cdot)$ is real-analytic in $U_{\delta}\left(p_{0}\right)$. The associated eigenfunction

$$
\Psi(\mu ; p, q, E(\mu, p))=\frac{C \mu \varphi(q)}{E(\mu, p)-w_{p}(q)}
$$

is analytic on $\mathbb{T}^{3}$, where $C \neq 0$ is a normalization constant. Moreover, the mappings

$$
\Psi: U_{\delta}\left(p_{0}\right) \rightarrow L^{2}\left(\mathbb{T}^{3}\right), \quad p \mapsto \Psi(\mu ; p, q, E(\mu, p)) \in L^{2}\left(\mathbb{T}^{3}\right)
$$

and

$$
\Psi:(\mu(p),+\infty) \rightarrow L^{2}\left(\mathbb{T}^{3}\right), \quad \mu \mapsto \Psi(\mu ; p, q, E(\mu, p)) \in L^{2}\left(\mathbb{T}^{3}\right)
$$

are vector-valued analytic functions in $U_{\delta}\left(p_{0}\right)$ and $(\mu(p),+\infty)$, respectively.

(ii) The operator $H_{\mu}(p)$ has no eigenvalues in a semi-infinite interval $(M(p), \infty)$ if and only if $0<\mu<\mu(p)$.

(iii) The threshold $z=M(p)$ is a regular point of the operator $H_{\mu}(p)$ if and only if $\mu \neq \mu(p)$. 
(iv) The threshold $z=M(p)$ is a virtual level of the operator $H_{\mu}(p)$ if and only if $\mu=\mu(p)$ and $\varphi\left(\mathbf{q}_{0}(p)\right) \neq 0$. The associated virtual state is of the form

$$
f(q)=\frac{C \mu(p) \varphi(q)}{M(p)-w_{p}(q)},
$$

where $C \neq 0$ is a normalizing constant and $f \in L^{1}\left(\mathbb{T}^{3}\right) \backslash L^{2}\left(\mathbb{T}^{3}\right)$.

(v) The threshold $z=M(p)$ is an eigenvalue of the operator $H_{\mu}(p)$ if and only if $\mu=\mu(p)$ and $\varphi\left(\mathbf{q}_{0}(p)\right)=0$. Moreover, if the threshold $z=M(p)$ is an eigenvalue of the operator $H_{\mu}(p)$, then the associated eigenfunction reads as

$$
f(q)=\frac{C \mu(p) \varphi(q)}{M(p)-w_{p}(q)} \in L^{2}\left(\mathbb{T}^{3}\right)
$$

where $C \neq 0$ is a normalizing constant.

Remark 4. It follows from the positivity of $\Phi^{*} \Phi$ that the operator $H_{\mu}(p)$ has no eigenvalues below $m(p)$.

\section{PROOF}

We begin with a series of auxiliary lemmata, which will be employed in the proof of the main result.

Lemma 3.1. Let Assumption 1 hold. Then there exist a $\delta$-neighborhood $U_{\delta}\left(p_{0}\right) \subset \mathbb{T}^{3}$ of the point $p=p_{0}$ and an analytic function $\mathbf{q}_{0}: U_{\delta}\left(p_{0}\right) \rightarrow \mathbb{T}^{3}$ such that for each $p \in U_{\delta}\left(p_{0}\right)$, the point $\mathbf{q}_{0}(p)$ is a unique non-degenerated maximum of the function $w_{p}(\cdot)$.

Proof. By Assumption 1, the square matrix

$$
A(0)=\left(\frac{\partial^{2} w_{p_{0}}}{\partial q_{i} \partial q_{j}}\left(\mathbf{q}_{0}\right)\right)_{i, j=1}^{3}<0
$$

is negatively defined and $\nabla w_{p_{0}}\left(q_{0}\right)=0$. Then by the implicit function theorem for the analytic case there exist a $\delta$-neighborhood $U_{\delta}\left(p_{0}\right) \subset \mathbb{T}^{3}$ of $p=p_{0} \in \mathbb{T}^{3}$ and a unique analytic vector function $\mathbf{q}_{0}(\cdot): U_{\delta}\left(p_{0}\right) \rightarrow \mathbb{T}^{3}$ such that $\nabla w_{p}\left(\mathbf{q}_{0}(p)\right)=0$ and

$$
A(p)=\left(\frac{\partial^{2} w_{p}}{\partial q_{i} \partial q_{j}}\left(\mathbf{q}_{0}(p)\right)\right)_{i, j=1}^{3}<0, \quad p \in U_{\delta}\left(p_{0}\right) .
$$

Hence, for each $p \in U_{\delta}\left(p_{0}\right)$, the point $\mathbf{q}_{0}(p)$ is a unique non degenerated maximum of the function $w_{p}(\cdot)$.

For each $\mu>0$ and $p \in \mathbb{T}^{3}$ we define in $\mathrm{C} \backslash[m(p) ; M(p)]$ an analytic function $\Delta(\mu, p ; \cdot)$ (the Fredholm determinant $\Delta(\mu, p ; \cdot)$ associated with the operator $\left.H_{\mu}(p)\right)$ :

$$
\Delta(\mu, p ; \cdot)=1-\mu \Omega(p ; \cdot)
$$

where

$$
\Omega(p ; z)=\int_{\mathbb{T}^{3}} \frac{\varphi^{2}(s) d s}{z-w_{p}(s)}, \quad p \in \mathbb{T}^{3}, \quad z \in \mathrm{C} \backslash[m(p) ; M(p)] .
$$

Lemma 3.2. A number $z \in \mathrm{C} \backslash \sigma_{\text {ess }}\left(H_{\mu}(p)\right), p \in \mathbb{T}^{3}$ is an eigenvalue of the operator $H_{\mu}(p)$ if and only if $\Delta(\mu, p ; z)=0$. The associated eigenfunction $f \in L^{2}\left(\mathbb{T}^{3}\right)$ reads as

$$
f(q)=\frac{C \mu \varphi(q)}{z-w_{p}(q)}
$$

where $C \neq 0$ is a normalizing constant. 
Proof. If a number $z \in \mathbb{C} \backslash \sigma_{\text {ess }}\left(H_{\mu}(p)\right), p \in \mathbb{T}^{3}$ is an eigenvalue of the operator $H_{\mu}(p)$ and $f \in L^{2}\left(\mathbb{T}^{3}\right)$ is an associated eigenfunction, i.e., the equation

$$
\left[w_{p}(q)-z\right] f(q)-\mu \varphi(q) \int_{\mathbb{T}^{3}} \varphi(t) f(t) d t=0,
$$

with

$$
\int_{\mathbb{T}^{3}} \varphi(t) f(t) d t \neq 0
$$

has solution, then the solution $f$ of equation 3.4 is given by

$$
f(q)=\frac{C \mu \varphi(q)}{z-w_{p}(q)}
$$

where $C \neq 0$ is a normalizing constant. The representation 3.5 of the solution of equation (3.4) implies the identity $\Delta(\mu, p ; z)=0$.

Let $\Delta(\mu, p ; z)=0$ for some $z \in \mathbb{C} \backslash \sigma_{e s s}\left(H_{\mu}(p)\right), p \in \mathbb{T}^{3}$. Then the function $f$ defined by (3.5) belong to $L^{2}\left(\mathbb{T}^{3}\right)$ and solves the equation

$$
H_{\mu}(p) f=z f .
$$

The analyticity of the eigenfunction $f(\cdot)$ defined by $(3.5)$ is implied by the analyticity of $\varphi(\cdot)$ and $w_{p}(\cdot)$ as well as by to the fact that the denominator $z-w_{p}(\cdot)$ in $(3.5)$ is non-zero.

Lemma 3.3. For $\zeta<0$ the following identities hold:

$$
I_{n}(\zeta)=\int_{0}^{\delta} \frac{r^{2 n} d r}{r^{2}-\zeta}=\frac{\pi}{2} \cdot \frac{\zeta^{n}}{\sqrt{-\zeta}}+\tilde{I}_{n}(\zeta), \quad n=0,1,2, \cdots
$$

where $\tilde{I}_{n}(\zeta)$ is an analytic function in a neighborhood of the origin.

The proof of the above lemma can be found in [20, Lm. 5].

Lemma 3.4. Let Assumption 1 hold. Then for each $p \in U_{\delta}\left(p_{0}\right)$ the integral

$$
\Omega(p)=\Omega(p, M(p))=\int_{\mathbb{T}^{3}} \frac{\varphi^{2}(s) d s}{M(p)-w_{p}(s)}
$$

is well-defined and is an analytic function in $U_{\delta}\left(p_{0}\right)$.

Proof. We note that by the parametrical Morse lemma [21, Lm. 3.3113], for each $p \in U_{\delta}\left(p_{0}\right)$, there exists a map $s=\psi(y, p)$ of the sphere $W_{\gamma}(0) \subset \mathbb{R}^{3}$ centered at $y=0$ of radius $\gamma>0$ into a neighborhood $U\left(\mathbf{q}_{0}(p)\right)$ of the point $\mathbf{q}_{0}(p)$ such that in $U\left(\mathbf{q}_{0}(p)\right)$, the function $w_{p}(\psi(y, p))$ can be represented as

$$
w_{p}(\psi(y, p))=M(p)-y_{1}^{2}-y_{2}^{2}-y_{3}^{2}=M(p)-y^{2} .
$$

Here the function $\psi(y, \cdot)$ (respectively, $\psi(\cdot, p)$ ) is analytic in $U_{\delta}\left(p_{0}\right)$ (respectively, $W_{\gamma}(0)$ ) and $\psi(0, p)=\mathbf{q}_{0}(p)$. Moreover, the Jacobian $J(\psi(y, p))$ of the mapping $s=\psi(y, p)$ is analytic in $W_{\gamma}(0)$ and positive, i.e., $J(\psi(y, p))>0$ for all $y \in W_{\gamma}(0)$ and $p \in U_{\delta}\left(p_{0}\right)$.

We represent

$$
\begin{aligned}
\Omega_{1}(p, z) & =\int_{\mathbb{T}^{3}} \frac{\varphi^{2}(s) d s}{z-w_{p}(s)}=\int_{U\left(\mathbf{q}_{0}(p)\right)} \frac{\varphi^{2}(s) d s}{z-w_{p}(s)}+\int_{\mathbb{T}^{3} \backslash U\left(\mathbf{q}_{0}(p)\right)} \frac{\varphi^{2}(s) d s}{z-w_{p}(s)} \\
& =\Omega_{1}(p, z)+\Omega_{2}(p, z) .
\end{aligned}
$$

Assumption 1 yields that the function $\Omega_{2}(p, M(p))$ is analytic in $p \in U_{\delta}\left(p_{0}\right)$. 
We change the variables $s=\psi(y, p)$ in the integral for $\Omega_{1}(p, z)$ and this gives

$$
\Omega_{1}(p, z)=\int_{W_{\gamma}(0)} \frac{\varphi^{2}(\psi(y, p))}{y^{2}+z-M(p)} J(\psi(y, p)) d y,
$$

where $J(\psi(y, p))$ is the Jacobian of the mapping $\psi(y, p)$. Passing to spherical coordinates as $y=r \nu$, we obtain

$$
\Omega_{1}(p, z)=\int_{0}^{\gamma} \frac{r^{2}}{r^{2}+z-M(p)}\left\{\int_{\Omega_{3}} \varphi^{2}(\psi(r \nu, p)) J(\psi(r \nu, p)) d \nu\right\} d r
$$

where $\Omega_{3}$ is a unit sphere in $\mathbb{R}^{3}$ and $d \nu$ is its area differential. The inner integral can be represented as

$$
\int_{\Omega_{3}} \varphi^{2}(\psi(r \nu, p)) J(\psi(r \nu, p)) d \nu=\sum_{n=0}^{\infty} \tau_{n}(p) r^{2 n}
$$

where the Pizetti coefficients $\tau_{n}(p), n=0,1,2, \ldots$ are analytic in $U_{\delta}\left(p_{0}\right)$ [19].

Identities (3.7) and (3.8) yield that the limit

$$
\Omega_{1}(p)=\lim _{z \rightarrow M(p)+0} \Omega_{1}(p, z)=\lim _{z \rightarrow M(p)+0} \sum_{n=0}^{\infty} \tau_{n}(p) \int_{0}^{\gamma} \frac{r^{2 n+2} d r}{r^{2}+z-M(p)}=\sum_{n=0}^{\infty} \frac{\gamma^{2 n+1}}{2 n+1} \tau_{n}(p)
$$

exists and the function $\Omega(p)=\Omega_{1}(p)+\Omega_{2}\left(p, M(p)\right.$ is analytic in $p \in U_{\delta}\left(p_{0}\right)$.

Lemma 3.5. Let Assumption 1 hold and $p \in U_{\delta}\left(p_{0}\right)$. Then the following statements are equivalent:

(i) the threshold $M(p)$ is a resonance of the operator $H_{\mu}(p)$ and the associated resonance state is of the form

$$
f(q)=\frac{C \mu(p) \varphi(q)}{M(p)-w_{p}(q)}
$$

where $C \neq 0$ is a normalizing constant.

(ii) $\varphi\left(\mathbf{q}_{0}(p)\right) \neq 0$ and $\Delta(\mu, p ; M(p))=0$.

(iii) $\varphi\left(\mathbf{q}_{0}(p)\right) \neq 0$ and $\mu=\mu(p)$.

Proof. Let the threshold $M(p)$ be a resonance of the operator $H_{\mu}(p)$. According to the definition of resonance, the equation

$$
H_{\mu}(p) f=M(p) f
$$

has a nontrivial solution $f \in L^{1}\left(\mathbb{T}^{3}\right) \backslash L^{2}\left(\mathbb{T}^{3}\right)$, i.e., the equation

$$
\left[M(p)-w_{p}(q)\right] f(q)-\mu \varphi(q) \int_{\mathbb{T}^{3}} \varphi(t) f(t) d t=0
$$

with

$$
\int_{\mathbb{T}^{3}} \varphi(t) f(t) d t \neq 0
$$

has a nontrivial solution. It is easy to check that the solution $f$ of equation (3.10), i.e., the virtual state, is given by (3.9). 
Expanding the function $\varphi(\psi(r \nu, p))$ in (3.7) to the Taylor series at $r=0$, we obtain

$$
\begin{aligned}
\varphi(\psi(r \nu, p))= & \varphi\left(\mathbf{q}_{0}(p)\right)+\sum_{i=1}^{3} \frac{\partial \varphi}{\partial \psi^{(i)}}\left(\mathbf{q}_{0}(p)\right)\left(\sum_{j=1}^{3} \frac{\partial \psi^{(i)}}{\partial y_{j}}(0, p) \nu_{j}\right) r \\
& +g(r, \nu) r^{2}, \quad y_{j}=r \nu_{j},
\end{aligned}
$$

where $g(\cdot, \nu)$ is continuous in $W_{\gamma}(0)$ and $\nu_{1}^{2}+\nu_{2}^{2}+\nu_{3}^{2}=1$. Since the solution $f$ of the equation (3.10) belongs to $L^{1}\left(\mathbb{T}^{3}\right) \backslash L^{2}\left(\mathbb{T}^{3}\right)$, asymptotics (3.11) yields the relation $\varphi\left(\mathbf{q}_{0}(p)\right) \neq 0$.

Substituting expression (3.9) for $f$ into equation (3.10), we obtain:

$$
\varphi(q)-\mu \varphi(q) \int_{\mathbb{T}^{3}} \frac{\varphi^{2}(t) d t}{M(p)-w_{p}(t)}=0,
$$

which implies the identities $\Delta(\mu, p ; M(p))=0$ and $\mu=\mu(p)$.

Let $\varphi\left(\mathbf{q}_{0}(p)\right) \neq 0$ and $\mu=\mu(p)$. Then it easy to confirm that $\Delta(\mu, p ; M(p))=0$ and the function $f$ defined by $(3.9)$ belongs to $L^{1}\left(\mathbb{T}^{3}\right) \backslash L^{2}\left(\mathbb{T}^{3}\right)$ and solves the equation

$$
H_{\mu}(p) f=M(p) f \text {. }
$$

Lemma 3.6. Let Assumption 1 hold and $p \in U_{\delta}\left(p_{0}\right)$. Then the following statements are equivalent:

(i) The threshold $z=M(p)$ is an eigenvalue of the operator $H_{\mu}(p)$ and the associated eigenvector is of the form

$$
f(q)=\frac{C \mu(p) \varphi(q)}{M(p)-w_{p}(q)}
$$

where $C \neq 0$ is a normalizing constant.

(ii) $\varphi\left(\mathbf{q}_{0}(p)\right)=0$ and $\Delta(\mu, p ; M(p))=0$.

(iii) $\varphi\left(\mathbf{q}_{0}(p)\right)=0$ and $\mu=\mu(p)$.

Proof. Let $z=M(p)$ be an eigenvalue of the operator $H_{\mu}(p)$ and $f \in L^{2}\left(\mathbb{T}^{3}\right)$ is an associated eigenfunction, i.e., the equation

$$
\left[M(p)-w_{p}(q)\right] f(q)-\mu \varphi(q) \int_{\mathbb{T}^{3}} \varphi(t) f(t) d t=0
$$

with

$$
\int_{\mathbb{T}^{3}} \varphi(t) f(t) d t \neq 0
$$

has a nontrivial solution. Then the associated eigenfunction $f$ is given by (3.13). In this case the relation $f \in L^{2}\left(\mathbb{T}^{3}\right)$ and asymptotics 3.11$)$ yield the the identity $\varphi\left(\mathbf{q}_{0}(p)\right)=0$. Equation (3.14) implies the identity $\Delta(\mu, p ; M(p))=0$, which implies $\mu=\mu(p)$.

Let $\varphi\left(\mathbf{q}_{0}(p)\right)=0$ and $\mu=\mu(p)$. Then $\Delta(\mu, p ; M(p))=0$ and the function $f$ defined by (3.13) solves the equation

$$
H_{\mu}(p) f=M(p) f
$$

Corollary 1. The equation $H_{\mu}(p) f=M(p) f$ has only trivial solution $f=0$ in $L^{2}\left(\mathbb{T}^{3}\right)$ if and only if $\mu \neq \mu(p)$.

In the next lemma we find an expansion for $\Delta(\mu, p ; z)$ in a half-neighborhood $(M(p), M(p)+\delta)$ of the point $z=M(p)$. 
Lemma 3.7. Let Assumption 1 hold. Then for each $\mu>0, p \in U_{\delta}\left(p_{0}\right)$ and sufficiently small $z-M(p)>0$ the function $\Delta(\mu, p ; \cdot)$ can be represented as the following convergent series:

$$
\begin{aligned}
& \Delta(\mu, p ; z)=1-\mu \Omega(p)+\mu \frac{\pi \tau_{0}(p)}{2}(z-M(p))^{1 / 2}-\mu \sum_{n=2}^{\infty} c_{n}(p)(z-M(p))^{n / 2}, \\
& \tau_{0}(p)=\varphi^{2}\left(\mathbf{q}_{0}(p)\right) J\left(\mathbf{q}_{0}(p)\right) .
\end{aligned}
$$

Proof. According to (3.7), (3.8) and Lemma 3.3, the function $\Omega_{1}(p ; z)$ can be represented as

$$
\Omega_{1}(p, z)=-\frac{\pi \tau_{0}(p)}{2}(z-M(p))^{1 / 2}+\sum_{n=1}^{\infty} \tilde{c}_{n}(p)(z-M(p))^{n+1 / 2}+\tilde{F}(p, z),
$$

where $\tilde{F}(p, z)$ is an analytic function at the point $z=M(p)$ and

$$
\tilde{c}_{n}(p)=\frac{(-1)^{n+1} \pi \tau_{n}(p)}{2} .
$$

Therefore, decomposition (3.6) yields the following representation for $\Omega(p, z), z \in$ $[M(p), M(p)+\delta):$

$$
\Omega(p, z)=-\frac{\pi \tau_{0}(p)}{2}(z-M(p))^{1 / 2}+\sum_{n=1}^{\infty} \tilde{c}_{n}(p)(z-M(p))^{n+\frac{1}{2}}+F(p, z),
$$

where $F(p, z)=\tilde{F}(p, z)+\Omega_{2}(p, z)$ is a function analytic at the point $z=M(p)$.

Since

$$
F(p, M(p))=\Omega(p) \quad \text { and } \quad(z-M(p))^{1 / 2}>0 \quad \text { for } \quad z>M(p)
$$

we obtain

$$
\Omega(p, z)=\Omega(p)-\frac{\pi \tau_{0}(p)}{2}(z-M(p))^{1 / 2}+\sum_{n=2}^{\infty} c_{n}(p)(z-M(p))^{n / 2} .
$$

Identity 3.1 completes the proof.

Now we are in position to prove our main result.

Proof of Theorem 2.1. We begin with proving (i). Let $\mu>\mu(p)$. Then Lemma 3.7 implies that

$$
\lim _{z \rightarrow M(p)+0} \Delta(\mu, p ; z)=\Delta(\mu, p ; M(p))=1-\frac{\mu}{\mu(p)}<0 .
$$

The function $\Delta(\mu, p ; \cdot)$ is continuous and monotonically increasing in $z \in(M(p),+\infty)$ and

$$
\lim _{z \rightarrow+\infty} \Delta(\mu, p ; z)=1 \text {. }
$$

Hence, $\Delta(\mu, p ; z)=0$ for a unique $z \in(M(p),+\infty)$.

Let $\Delta(\mu, p ; z)=0$ for some $z \in(M(p),+\infty)$. Then

$$
1-\frac{\mu}{\mu(p)}=\Delta(\mu, p ; M(p))<\Delta(\mu, p ; z)=0
$$

which yields that $\mu>\mu(p)$. Applying Lemma 3.6, we arrive at the desired statement.

Since $z=E(\mu, p)$ is a solution of the equation $\Delta(\mu, p ; z)=0$ and $\Delta(\mu, \cdot ; z)(\operatorname{resp} . \Delta(\cdot, p ; z))$ is real-analytic in $U_{\delta}\left(p_{0}\right)$ (resp. $\left.(\mu(p),+\infty)\right)$, the implicit function theorem implies that $E(\mu, \cdot)$ (respectively, $E(\cdot, p)$ ) is real analytic in $U_{\delta}\left(p_{0}\right)$ (respectively, $(\mu(p),+\infty)$ ).

Note that for each $p \in U_{\delta}\left(p_{0}\right)$ the determinant $\Delta(\cdot, p ; z)$ is monotonically decreasing function in $(\mu(p),+\infty)$ and hence, the solution (eigenvalue) $E(\mu, p)$ is also monotonically decreasing function in $(\mu(p),+\infty)$. 
Lemma 3.2 implies that if a number $E(\mu, p)$ is an eigenvalue of $H_{\mu}(p), p \in U_{\delta}\left(p_{0}\right)$, then the function

$$
\Psi(\mu ; p, \cdot, E(\mu, p))=\frac{C \mu \varphi(\cdot)}{E(\mu, p)-w_{p}(\cdot)},
$$

where $C \neq 0$ is a normalization constant, is a solution of the equation

$$
H_{\mu}(p) \Psi(\mu ; p, q, E(\mu, p))=E(\mu, p) \Psi(\mu ; p, q, E(\mu, p)) .
$$

The analyticity of $\Psi(\mu ; p, \cdot, E(\mu, p))$ follows the analyticity of $\varphi(\cdot)$ and $\left(w_{p}(\cdot)-E(\mu, p)\right)^{-1}$ in $\mathbb{T}^{3}$.

Since the functions $E(\mu, \cdot)$ (respectively, $E(\cdot, p)$ ) and $w(\cdot, q)$ are analytic in $U_{\delta}\left(p_{0}\right)$ (respectively, $(\mu(p),+\infty))$ and $w_{p}(q)-E(\mu, p)>0$ the mapping $p \mapsto \Psi(\mu ; p, q, E(\mu, p))$ (respectively, $\mu \mapsto \Psi(\mu ; p, q, E(\mu, p)))$ is also analytic mapping in $U_{\delta}\left(p_{0}\right)$ (respectively, $(\mu(p),+\infty)$ ).

The rest of statements of Theorem 2.1 can be proven by applying Lemmata 3.5 and 3.6 in the same way as in the above proof of $(i)$.

\section{ACKNOWLEDGMents}

Authors thank the referees for a number of constructive comments and useful suggestions.

\section{BIBLIOGRAPHY}

1. S.N. Lakaev, A. Ibrahim, Sh.H. Kurbanov. Threshold effects for the generalized Friedrichs model with the perturbation of rank one // Abstr. Appl. Anal. 2012, 180953 (2012).

2. S.N. Lakaev. The stucture of resonances of the Generalized Friedrichs model // Funkts. Anal. Pril. 17:4, 88-89(1983). [Funct. Anal. Appl. 17:4, 317-319 (1983).]

3. S.N. Lakaev. Some spectral properties of the Generalized Friedrichs model // Trudy Sem. Petrovsk. 11, 210-238 (1986). [J.Soviet Math. 45:6, 1540-1565 (1989).]

4. E.L. Lakshtanov, R.A. Minlos. The spectrum of two-particle bound states of transfer matrices of Gibbs fields (an isolated bound state) // Funkts. Anal. Prilozhen. 38:3, 52-69(2004). [Funct. Anal. Appl. 38:3, 202-216 (2004).]

5. J. Hecker Denschlag, A.J. Daley. Exotic atom pairs: Repulsively bound states in an optical lattice // in "Proceedings of the International School of Physics "Enrico Fermi"". 164. 677-696 (2007).

6. K. Winkler, G. Thalhammer, F. Lang, R. Grimm, J. Hecker Denschlag, A. J. Daley, A. Kantian, H. P. Bc̈hler, P. Zoller. Repulsively bound atom pairs in an optical lattice. // Nature. 441, 853-856 (2006).

7. Zh.I. Abdullaev, I.A. Ikromov, S.N. Lakaev. Embedded eigenvalues and resonances of a generalized Friedrichs model // Teor. Matem. Fiz. 103:1, 54-62 (1995). [Theor. Math. Phys. 103:1, 390-397 (1995).]

8. A. Mogilner. Hamiltonians in solid state physics as multi-particle discrete Schrödinger operators: Problems and results // Adv. Soviet Math. 5, 139-194 (1991).

9. M. Reed, B. Simon. Methods of modern mathematical physics. III: Scattering theory. Academic Press, New York (1979).

10. K.O. Friedrichs. On the perturbation of continuous spectra // Comm. Appl. Math. 1:4, 361-406 (1948).

11. K.O. Friedrichs. Perturbation of spectra in Hilbert space, Amer. Math. Soc., Providence, RI (2008).

12. V.A. Malishev, R.A. Minlos. Linear infinite-particle operators. Transl. Math. Monogr. 143. Amer. Math. Soc., Providence, RI (1995).

13. S. Albeverio, S.N. Lakaev, Z.I. Muminov. The threshold effects for a family of Generalized Friedrichs models under rank one perturbations // J. Math. Anal. Appl. 330:2, 1152-1168 (2007).

14. S.N. Lakaev, M. Darus, Sh.H. Kurbanov. Puiseux series expansion for an eigenvalue of the generalized Friedrichs model with perturbation of rank 1 // J. Phys. A: Math. Theor. 46:20, 205304 (2013). 
15. M. Klaus, B. Simon. Coupling constant thresholds in nonrelativistic quantum mechanics. I. Shortrange two-body case // Ann. Phys. 130:2, 251-281 (1980).

16. S.N. Lakaev, Sh.Yu. Holmatov. Asymptotics of Eigenvalues of a two-particle Schrödinger operators on lattices with zero range interaction // J. Phys. A: Math. Theor. 44:13, 135304 (2011).

17. S. Albeverio, S.N. Lakaev, K.A. Makarov, Z.I. Muminov. The threshold effects for the two-particle Hamiltonians on lattices // Comm. Math. Phys. 262:1, 91-115 (2006).

18. M. Reed, B. Simon. Methods of modern mathematical physics. IV: Analysis of operators, Academic Press, New York (1978).

19. R. Courant, D. Hilbert. Methods of mathematical physics. V. 2. Partial differential equations, John Wiley \& Sons, New York (1966).

20. S.N. Lakaev. Bound states and resonances of $N$-particle discrete Schrödinger operator // Theor. Matem. Fiz. 91:1, 51-65(1992). [Theor. Math. Phys. 91:1, 362-372 (1992).]

21. M.V. Fedoryuk. Asymptotics of integrals and series, Nauka, Moscow (1987). (in Russian).

Saidakhmat Norjigitovich Lakaev,

Samarkand State University,

University Boulvard 15,

140104, Samarkand, Uzbekistan

E-mail: slakaev@mail.ru

Maslina Darus,

University Kebangsaan Malaysia,

43600, Selangor, Malaysia

E-mail: maslina@ukm.my

Said T. Dustov,

Navoi State Pedagogical Institute,

Janubiy 1-A, Navoi,

210102, Navoi, Uzbekistan

E-mail: SaidDustov@mail.ru 